\title{
Effect of oxygen plasma treatment on the structure and mechanical properties of bilayer graphene studied by molecular dynamics simulation
}

Jincong Chen ${ }^{\dagger,}$, , Jiayun Pei ${ }^{\dagger}$, , Haiyan Zhao ${ }^{\dagger},, *$

${ }^{\dagger}$ Department of Mechanical Engineering, Tsinghua University, Beijing 100084, PR China

${ }^{\S}$ State Key Laboratory of Tribology, Tsinghua University, Beijing 100084, PR China

Figure S1 shows typical stress-strain curves of uniaxial stretching of bilayer graphene with different number of oxygen atoms.

(a)

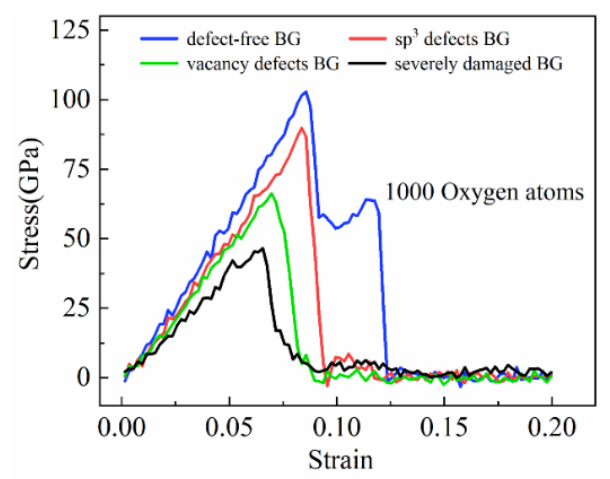

(c)

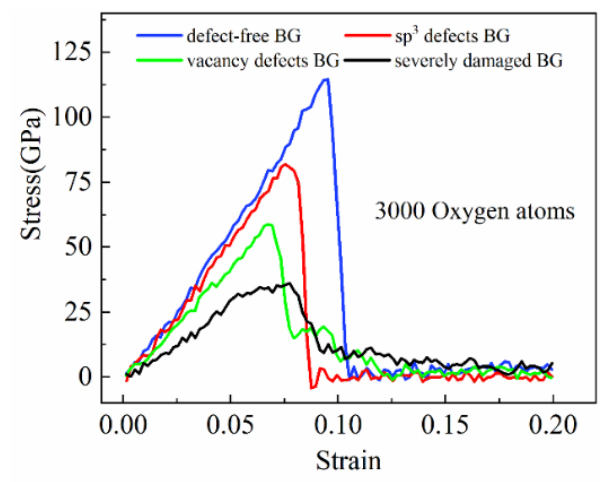

(b)

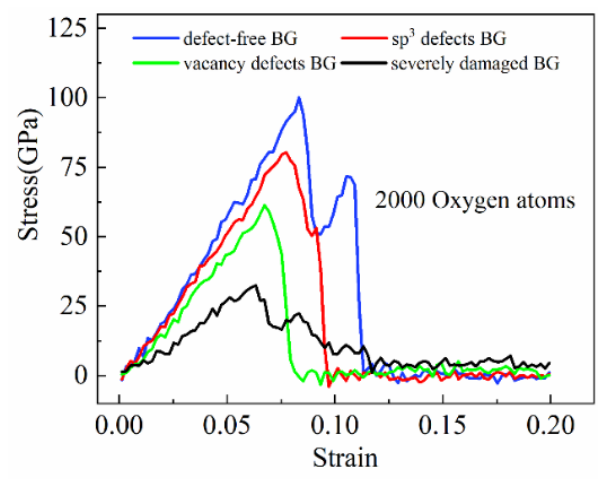

(d)

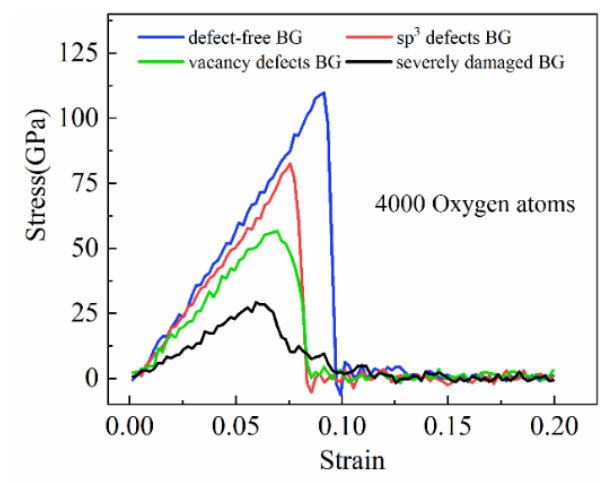

Figure S1. Typical stress-strain curves of uniaxial stretching of bilayer graphene with (a) 1000 oxygen atoms, (b) 2000 oxygen atoms, (c) 3000 oxygen atoms and (d) 4000 oxygen atoms. 
We selected 4 typical structures: defect-free bilayer graphene close to forming $\mathrm{sp}^{3}$ defects, bilayer graphene with only some $\mathrm{sp}^{3}$ defects, bilayer graphene with few vacancy defects and severely damaged bilayer graphene. The stress-strain curves corresponding to these 4 structures have been drawn, as shown in Fig. S1. The four sub-figures correspond to four different numbers of oxygen atoms. 